\section{Atividade da butirilcolinesterase e fatores de risco cardiovascular em adolescentes obesos submetidos a um programa de exercícios físicos}

\author{
Butyrylcholinesterase activity and cardiovascular risk factors \\ in obese adolescents submitted to an exercise program
}

Gerusa Eisfeld Milano', Neiva Leite', Thais Januzzi Chaves², Gisele Eisfeld Milano ${ }^{2}$, Ricardo Lehtonen Rodrigues de Souza ${ }^{2}$, Lupe Furtado Alle ${ }^{2}$

\section{RESUMO}

Objetivo: Avaliar o efeito de 12 semanas de exercícios físicos em variáveis associadas a fatores de risco cardiovascular e na atividade da butirilcolinesterase (BChE) em adolescentes obesos. Sujeitos e métodos: A amostra foi composta por 24 obesos e 51 eutróficos controles. Inicialmente e após 12 semanas foram avaliados: peso, estatura, IMC, circunferência abdominal (CA), percentual de gordura $(\% \mathrm{G})$, consumo máximo de oxigênio $\left(\mathrm{VO}_{2}\right.$ máx), pressão arterial sistólica (PAS) e diastólica (PAD), glicemia (GLI) e insulinemia (INS) basal e após $120 \mathrm{~min}$, triacilglicerol (TG), colesterol total (CT), colesterol LDL, colesterol HDL e a atividade da BChE (kU/l). Resultados: Após a intervenção, houve redução significativa no IMC, CA, \%G, PAD, PAD, TG, GLI 120, INS, INS 120 min e na atividade da $\mathrm{BChE}$. Conclusão: A redução da atividade da $\mathrm{BChE}$, observada após a intervenção, foi acompanhada da redução de variáveis associadas a risco cardiovascular e à obesidade, indicando que a BChE pode ser utilizada como marcador secundário para os riscos associados à obesidade precoce. Arq Bras Endocrinol Metab. 2013;57(7):533-7

\section{Descritores}

Exercício físico; atividade da BChE; obesidade precoce

\begin{abstract}
Objective: To evaluate the effect of 12 weeks of physical exercise (PE) on cardiovascular risk factors and BChE activity in obese adolescents. Subjects and methods: The sample consisted of 24 obese adolescents and 51 normal weight controls. The following variables were measured in the initial stage and after 12 weeks: weight, height, BMI, waist circumference (WC), fat percentage (\% F), maximal oxygen uptake ( $\mathrm{VO}_{2} \mathrm{max}$ ), systolic (SBP) and diastolic (DBP) blood pressure, glucose (GLY) and insulin (INS) at baseline and after $120 \mathrm{~min}$, triacylglycerol (TG), total cholesterol (TC), LDL cholesterol, HDL cholesterol, and BChE activity (kU/l). Results: After the intervention, there was significant reduction in BMI, WC, \%F,TG, GLI 120, INS 120 min, and BChE activity. Conclusion: The reduction in BChE activity, observed after physical exercise, was accompanied by the reduction of the variables associated with cardiovascular risk and obesity, indicating that $\mathrm{BChE}$ can be used as a secondary marker for the risk associated with early onset obesity. Arq Bras Endocrinol Metab. 2013;57(7):533-7
\end{abstract}

Keywords

Physical exercise; BChE activity; early onset obesity
${ }^{1}$ Núcleo de Pesquisa em Qualidade de Vida (NOV), Universidade Federal do Paraná (UFPR), Departamento de Genética, Curitiba, PR, Brasil ${ }^{2}$ Laboratório de Polimorfismo e Ligação, UFPR, Departamento de Genética, Curitiba, PR, Brasil
Correspondência para:

Gerusa Eisfeld Milano

Departamento de Genética, Universidade Federal do Paraná

Rua Coronel Francisco Heráclito dos Santos, 210

81531-970 - Curitiba, PR, Brasil gerusapersonal@gmail.com

Recebido em 24/Out/2012 Aceito em 14/Abr/2013

\section{INTRODUÇÃO}

A prevalência de sobrepeso e obesidade tem aumentado nesta década, e o desafio para os profissionais da área da saúde está na prevenção e na terapêutica adotada para redução do excesso de peso. $\mathrm{O}$ interesse para o maior entendimento dos mecanismos envolvidos no controle ponderal cresceu principalmente pelo aumento na prevalência do excesso de peso em todas as faixas etárias $(1,2)$. 
Além de fatores ambientais, a análise mais aprofundada do papel desempenhado por enzimas relacionadas ao perfil lipídico também pode ser importante no entendimento dos processos lipolíticos (3). A enzima butirilcolinesterase (BChE) tem sido associada a alguns fatores de risco para doenças cardiovasculares, tais como: obesidade, metabolismo de lípides, pressão sanguínea e comportamento da insulina (4). Estudos realizados com obesos adultos compararam a atividade da BChE entre obesos e eutróficos e verificaram maiores valores na atividade da BChE em obesos $(5,6)$. Nesse contexto, é relevante a realização de estudo que avalie o comportamento da atividade da BChE em obesos após uma intervenção com exercício físico. Desse modo, o objetivo do presente estudo foi avaliar o efeito de 12 semanas de exercícios físicos (EF) sobre a atividade da BChE e sobre variáveis associadas a fatores de risco cardiovascular em adolescentes obesos.

\section{SUJEITOS E MÉTODOS}

\section{Participantes do estudo}

Participaram do estudo de intervenção 24 obesos, com idade entre 10 e 16 anos, de ambos os gêneros (12,06 \pm 2,01 anos). Para ter um valor do controle da atividade da $\mathrm{BChE}$, foram analisados 51 adolescentes eutróficos (18,82 $\pm 0,62$ anos). Os pais ou responsáveis dos participantes assinaram o Termo de Consentimento Livre e Esclarecido, aprovado pelo Comitê de Ética da Universidade Federal do Paraná (UFPR), que atende à Resolução nº 196/96.

\section{Avaliações antropométricas e clínicas}

A estatura foi avaliada em estadiômetro de parede, marca Ayrton Corporation ${ }^{\circledR}$, com precisão de $0,1 \mathrm{~cm}$, com o indivíduo em posição ortostática, com os pés descalços e unidos, com as superfícies posteriores do calcanhar, cinturas pélvica e escapular e região occipital em contato com o instrumento de medida, com a cabeça no plano horizontal de Frankfort, ao final de uma inspiração máxima. O peso foi avaliado em balança marca Filizola ${ }^{\circledR}$, tipo plataforma, com capacidade máxima de $150 \mathrm{~kg}$ e precisão de 100 gramas, com o indivíduo descalço, posicionado em pé no centro da plataforma, com os braços ao longo do corpo e utilizando roupas íntimas. O IMC, expresso em $\mathrm{kg}$ por $\mathrm{m}^{2}$, foi calculado por meio da razão do peso $(\mathrm{kg})$ pelo quadrado da estatura $\left(\mathrm{m}^{2}\right)$ e classificado conforme valores disponibi- lizados pelo CDC (7), para cada faixa etária e sexo. O IMC-escore $\mathrm{Z}$ foi calculado subtraindo-se o valor correspondente ao $50^{\circ}$ percentil do IMC de cada avaliado, dividindo-se pelo desvio-padrão populacional (7).

A circunferência abdominal (CA) foi mensurada em $\mathrm{cm}$, com uma fita flexível e inextensível, com precisão de $0,1 \mathrm{~cm}$, aplicada acima da crista ilíaca, paralela ao solo, com o indivíduo em pé, com o abdome relaxado e com os braços ao longo do corpo e os pés unidos.

Para determinação do percentual de gordura $(\% \mathrm{G})$, foi utilizado o método de impedância bioelétrica (BIA) tetrapolar, por aparelho Maltron modelo BF906, com o posicionamento dos eletrodos nas superfícies dorsais das mãos e pés direitos, próximos às articulações metacarpofalangianas e metatarsofalangianas e medialmente entre as proeminências distais do rádio e da ulna e entre o maléolo tibial e fibular.

As pressões arteriais sistólica (PAS) e diastólica (PAD) foram medidas no indivíduo sentado, após 10 minutos de repouso. A $\mathrm{FC}_{\text {rep }}$ foi obtida por meio de um frequencímetro da marca Polar. A pressão arterial (PA) será mensurada no braço direito apoiado em nível cardíaco, utilizando-se esfigmomanômetro de mercúrio, do tipo aneroide, previamente calibrado conforme o Inmetro, com o tamanho do manguito apropriado ao perímetro do braço do indivíduo.

\section{Exames laboratoriais}

As amostras sanguíneas foram coletadas no período da manhã, após 12 horas de jejum, para a realização de hemograma e dosagens de glicose, insulina, colesterol total (CT), HDL, LDL e triacilglicerol (TG).

As concentrações plasmáticas de CT, TG e de HDL-colesterol foram determinadas em $\mathrm{mg} / \mathrm{dL}$, e foi utilizado o teste colorimétrico enzimático (CHOD-PAP) (Laboratório Merck, Darmstadt, Alemanha; Laboratório Roche, Indianápolis, IN, EUA). O LDL-colesterol foi calculado pela fórmula de Friedewald, em mg/dL.

A insulina foi dosada pela técnica de quimiluminescência por imunoensaio imunométrico em $\mu \mathrm{U} / \mathrm{ml}$, em equipamento automatizado Immulite 2000.

Foi utilizada a técnica de Dietz e cols. (8), modificada por Evans e Wroe (9) para a determinação da atividade plasmática da $\mathrm{BChE}(\mathrm{kU} / \mathrm{l})$.

\section{Aptidão aeróbia $\left(\mathrm{VO}_{2}\right.$ máx $)$}

A avaliação cardiorrespiratória foi medida por meio das trocas gasosas com um sistema metabólico direto ( Vis- 
ta XT metabolic system, EUA) computadorizado (Intel 486, DX2, $66 \mathrm{mhz}$ ). Foi utilizado um conjunto de máscara, gorro e turbina com vedação da boca e nariz, levando o ar expirado ao equipamento de análise de gases. Para a monitoração da frequência cardíaca, foi utilizado um frequencímetro cardíaco (Polar - Al). O teste foi realizado em esteira ergométrica, e o protocolo utilizado foi o de Balke modificado, mantendo a velocidade fixa em 3,25 mph e inclinação de 6\%, com incremento de $2 \%$ a cada 3 minutos, até o esforço máximo (10).

Considerou-se $\mathrm{VO}_{2 \max }$ quando pelo menos dois dos seguintes critérios foram observados: a) exaustão ou inabilidade para manter a velocidade requerida; b) RER $>1,0$; c) FC > 190 bpm. Utilizaram-se as médias dos três maiores valores seguidos para o estabelecimento do $\mathrm{VO}_{2 \max }$.

\section{Programa de exercício físico}

O programa de exercício físico consistiu em 12 semanas de atividades aeróbias, e os exercícios eram praticados três vezes por semana, com cada sessão consistindo em $110 \mathrm{~min}$ (45 min de caminhada, $45 \mathrm{~min}$ de ciclismo indoor e 20 min de alongamento). A faixa de treinamento individualizada para a caminhada e o ciclismo indoor foi calculada a partir da frequência cardíaca máxima (FCmáx) e do $\mathrm{VO}_{2 \text { máx }}$ obtidos nos testes ergométricos. $\mathrm{O}$ ciclismo indoor e a caminhada iniciaram-se na intensidade entre $35 \%$ e $55 \%$ da FC de reserva (FCR), aumentando para $45 \%$ a $65 \%$, na $5^{\mathrm{a}}$ a $8^{\mathrm{a}}$ semana, atingindo entre $55 \%$ e $75 \%$ da FCR na $9^{\text {a }}$ a $12^{\text {a }}$ semana.

\section{Análise estatística}

Foram utilizados o programa Statistica para Windows para cálculos de médias e desvios-padrão e o teste $t$ de Student com nível de significância de $\mathrm{p}<0,05$.

\section{RESULTADOS}

Este estudo avaliou variáveis antropométricas e atividade da enzima BChE em 24 adolescentes com excesso de peso, sendo 13 meninas e 11 meninos com média de idade de 12,06 $\pm 2,01$ anos, assim como de 51 adolescentes eutróficos. Na tabela 1 encontram-se os dados iniciais do grupo obeso comparados aos do grupo eutrófico.

Na tabela 2 são apresentados os dados antropométricos e metabólicos do grupo antes e após a intervenção e os valores de p para o teste $\mathrm{t}$ de Student (Tabela 2).
Tabela 1. Médias e desvios-padrão das variáveis antropométricas e da atividade da BChE nos grupos de obesos pré-intervenção e de eutróficos e valores de t e p para comparação das médias entre os grupos

\begin{tabular}{lccc}
\hline & $\begin{array}{c}\text { Obesos } \\
\mathbf{M} \pm \mathbf{D P}\end{array}$ & $\begin{array}{c}\text { Eutróficos } \\
\mathbf{M} \pm \mathbf{D P}\end{array}$ & $\mathbf{p}$ \\
\hline Idade (anos) & $12,06 \pm 2,01$ & $18,82 \pm 0,62$ & $\mathrm{p}<0,05$ \\
Peso (kg) & $72,12 \pm 18,20$ & $68,62 \pm 6,70$ & $\mathrm{~ns}$ \\
Estatura (m) & $1,55 \pm 0,11$ & $1,75 \pm 0,6$ & $\mathrm{p}<0,05$ \\
IMC (kg/m²) & $29,25 \pm 4,67$ & $22,33 \pm 1,48$ & $\mathrm{p}<0,05$ \\
Atividade da BChE (kU/l) & $7,46 \pm 3,16$ & $4,41 \pm 0,78$ & $\mathrm{p}<0,05$ \\
\hline
\end{tabular}

Tabela 2. Médias e desvios-padrão dos dados pré e pós-intervenção e valores de $p$ para 0 teste $t$ de Student

\begin{tabular}{lccc}
\hline & $\begin{array}{c}\text { Inicial } \\
\text { Média } \pm \text { DP }\end{array}$ & $\begin{array}{c}\text { Final } \\
\text { Média } \pm \text { DP }\end{array}$ & p \\
\hline Estatura (m) & $1,55 \pm 0,11$ & $1,56 \pm 0,11$ & $\mathrm{p}<0,05$ \\
Peso (kg) & $72,12 \pm 18,20$ & $71,18 \pm 17,79$ & $\mathrm{~ns}$ \\
IMC (kg/m²) & $29,25 \pm 4,67$ & $28,53 \pm 4,63$ & $\mathrm{p}<0,05$ \\
Score Z IMC & $2,80 \pm 0,96$ & $2,62 \pm 0,84$ & $\mathrm{p}<0,05$ \\
\%G & $39,01 \pm 5,09$ & $38,01 \pm 5,17$ & $\mathrm{p}<0,05$ \\
CA (cm) & $96,36 \pm 11,49$ & $94,29 \pm 10,82$ & $\mathrm{p}<0,05$ \\
PAS (mmHg) & $109,72 \pm 15,48$ & $109,86 \pm 13,86$ & $\mathrm{~ns}$ \\
PAD (mmHg) & $72,40 \pm 11,39$ & $68,78 \pm 9,36$ & $\mathrm{~ns}$ \\
VO ${ }_{\text {2máx }}$ & $30,99 \pm 5,87$ & $34,23 \pm 6,02$ & $\mathrm{p}<0,05$ \\
FC ${ }_{\text {máx }}$ & $192,5 \pm 12,35$ & $191,07 \pm 9,39$ & $\mathrm{~ns}$ \\
CT (mg/dL) & $156,12 \pm 29,17$ & $155,95 \pm 29,58$ & $\mathrm{~ns}$ \\
HDL (mg/dL) & $46,08 \pm 8,30$ & $47 \pm 8,88$ & $\mathrm{~ns}$ \\
LDL (mg/dL) & $89,05 \pm 25,54$ & $93,95 \pm 26,06$ & $\mathrm{~ns}$ \\
TG (mg/dL) & $105,12 \pm 47,62$ & $79,08 \pm 33,54$ & $\mathrm{p}<0,05$ \\
Glicemia (mg/dL) & $90,79 \pm 7,24$ & $91,70 \pm 7,73$ & $\mathrm{~ns}$ \\
Glicemia 120 (mg/dL) & $94,08 \pm 18,98$ & $83,22 \pm 11,97$ & $\mathrm{p}<0,05$ \\
Ins. basal (mg/dL) & $15,75 \pm 12,05$ & $11,34 \pm 7,74$ & $\mathrm{~ns}$ \\
Ins. 120 min (mg/dL) & $94,08 \pm 30,69$ & $27,11 \pm 19,32$ & $\mathrm{p}<0,05$ \\
Ativ. BChE (kU/l) & $7,46 \pm 3,16$ & $5,54 \pm 1,93$ & $\mathrm{p}<0,05$ \\
\hline & & &
\end{tabular}

\section{DISCUSSÃO}

Com a prática de 12 semanas de exercício físico orientado, houve redução significativa no IMC, CA, \%G, PAS, PAD, TG, GLI 120, INS, INS 120 e atividade da BChE. Houve também aumento no $\mathrm{VO}_{2 \text { máx }}$, demonstrando que o grupo submetido ao treinamento teve melhoras no condicionamento cardiorrespiratório.

$\mathrm{Na}$ literatura já está demonstrado que a prática de atividades aeróbias em intensidades de moderadas a intensa na infância e adolescência, praticadas duas ou mais vezes na semana, com duração de no mínimo seis semanas, melhorou o perfil lipídico e antropométrico $(11,12)$. Dessa forma, a escolha da intensidade, 
frequência e duração do treinamento utilizadas neste estudo está de acordo com pesquisas já realizadas.

Os trabalhos que utilizam tratamento não medicamentoso com a realização de exercício de forma isolada ou associado à orientação nutricional demonstram uma variedade de resultados no que se refere às modificações no perfil metabólico. No presente estudo foram encontradas reduções em TG, glicemia de jejum e INS120 min, concordando parcialmente com outros estudos que encontraram redução no CT (13-16), no LDL $(13,16)$ no TG $(16,17)$ e na INS $(16)$.

A BChE relaciona-se com o metabolismo de lípides e também com fatores que afetam a obesidade, como IMC, triglicérides, lipoproteínas, entre outros como a proliferação celular, o crescimento neural e a adesão celular. Os valores elevados da atividade da enzima BChE encontrados na amostra de adolescentes obesos concordam com estudos anteriores que mostram maiores valores da atividade da $\mathrm{BChE}$ em indivíduos adultos obesos quando comparados aos não obesos $(5,6,18)$.

No presente estudo a atividade enzimática no grupo obeso é significativamente maior que em não obesos $(7,46 \pm 3,16$ e $4,9 \pm 0,1$, respectivamente; $\mathrm{p}<0,05)$, porém, após o tratamento com exercício físico, os valores da atividade da BChE tornaram-se semelhantes àqueles verificados em indivíduos eutróficos $(5,54 \pm$ $1,93$ e 4,9 $\pm 0,1$, respectivamente; $\mathrm{p}>0,05)$. Como já sugerido por Kalman e cols. (19), o aumento na atividade da BChE pode ser consequência de distúrbios associados à obesidade, como mostra a diminuição concomitante da atividade enzimática, IMC e triglicerídeos. Estudos anteriores (20-22) sugerem que o papel da BChE no metabolismo de lipídios seja a hidrólise de ésteres de colina, que são produtos do metabolismo de ácidos graxos livres e da lipogênese hepática. Desse modo, um aumento na disponibilidade e/ou do fluxo hepático de ácidos graxos livres ou um aumento da lipogênese a partir de carboidratos poderia levar a dois efeitos: hiperlipidemia e aumento da atividade da BChE. Assim, a BChE pode estar ligada indiretamente a doenças metabólicas, pois, além do decréscimo da sua atividade, também foi verificada redução significativa de fatores de risco (IMC, triglicerídeos e circunferência abdominal).

O presente estudo permite reforçar as hipóteses de associação da BChE com a obesidade e o metabolismo de lipídios, mostrando que essa associação está presente também na obesidade precoce. Embora não seja um fator de risco para o excesso de peso, pois existem outros fatores metabólicos e genéticos envolvidos na sua regulação, a atividade da BChE está relacionada à obesidade precoce e, assim como outras variáveis associadas, responde à prática de exercícios físicos e pode ser utilizada como marcador secundário para os riscos associados à obesidade precoce.

Agradecimentos: este estudo foi parcialmente financiado pela Fundação Araucária. A primeira autora é bolsista da Coordenação de Aperfeiçoamento de Pessoal de Nível Superior (Capes) e a última autora é bolsista PQ do Conselho Nacional de Desenvolvimento Científico e Tecnológico (CNPq).

Declaração: os autores declaram não haver conflitos de interesse científico neste estudo.

\section{REFERÊNCIAS}

1. Janssen I, Katzmarzyk PT, Boyce WF, Vereecken C, Mulvihill C, Roberts $\mathrm{C}$, et al. Comparison of overweight and obesity prevalence in school-aged youth from 34 countries and their relationships with physical activity and dietary patterns. Obes Rev. 2005;6(2):123-32.

2. Rodrigues AM, Suplicy HL, Radominski RB. Controle neuroendócrino do peso corporal: implicações na gênese da obesidade. Arq Bras Endocrinol Metab. 2003;47(4):398-409.

3. Mattevi VS, Zembrzuski VM, Hutz MH. Impact of variation in ADRB2, ADRB3, and GNB3 genes on body mass index and waist circumference in a Brazilian population. Am J Hum Biol. 2006;18(2):182-6.

4. Benyamin B, Middelberg RP, Lind PA, Valle AM, Gordon S, Nyholt $D R$, et al. GWAS of butyrylcholinesterase activity identifies four novel loci, independent effects within BCHE and secondary associations with metabolic risk factors. Hum Mol Genet. 2011;20(22):4504-14.

5. Furtado-Alle L, Andrade FA, Nunes K, Mikami LR, Souza RL, Chautard-Freire-Maia EA. Association of variants of the -116 site of the butyrylcholinesterase BCHE gene to enzyme activity and body mass index. Chem Biol Interact. 2008;175(1-3):115-8.

6. Boberg DR, Furtado-Alle L, Souza RL, Chautard-Freire-Maia EA. Molecular forms of butyrylcholinesterase and obesity. Genet Mol Biol. 2010;33(3):452-4.

7. Kuczmarski RJ, Ogden CL, Grummer-Strawn LM, Flegal KM, Guo SS, Wei R, et al. CDC growth charts: United States. Adv Data. 2000;(314):1-27.

8. Dietz AA, Rubinstein HM, Lubrano T, Hodges LK. Improved method for the differentiation of cholinesterase variants. Am J Hum Genet. 1972;24(1):58-64.

9. Evans RT, Wroe J. Is serum cholinesterase activity a predictor of succinyl choline sensitivity? An assessment of four methods. Clin Chem. 1978;24(10):1762-6.

10. Rowland TW. Exercise and children's health. Champaign: Human Kinetics Books; 1990.

11. Leite N, Milano GE, Cieslak F, Lopes WA, Rodacki A, Radominski RB. Efeito do exercício físico e da orientação nutricional na síndrome metabólica em adolescentes obesos. Rev Bras Fisioter. 2009;13(1):73-81.

12. Kang HS, Gutin B, Barbeau P, Owens S, Lemmon CR, Allison J, et al. Physical training improves insulin resistance syndrome markers in obese adolescents. Med Sport Sci. 2002;34(12):1920-7. 
13. Nemet D, Barkan S, Epstein Y, Friedland O, Kowen G, Eliakim A. Short- and long-term beneficial effects of a combined dietarybehavioral-physical activity intervention for the treatment of childhood obesity. Pediatr. 2005;115(4):e443-9.

14. Sabia RV, Santos JE, Ribeiro RPP. Efeito da atividade física associada à orientação alimentar em adolescentes obesos: comparação entre exercício aeróbio e anaeróbio. Rev Bras Med Esport. 2004;10(5):349-55.

15. Parente EB, Guazzelli I, Ribeiro MM, Silva AG, Halpern A, Villares SM. Perfil lipídico em crianças obesas: efeitos de dieta hipocalórica e atividade física aeróbica. Arq Bras Endocrinol Metab. 2006;50(3):499-504.

16. Park $\mathrm{T}$, Hong $\mathrm{H}$, Lee $\mathrm{J}$, Kang $\mathrm{H}$. Lifestyle plus exercise intervention improve metabolic syndrome markers without change in adiponectin in obese girls. Ann Nutr Metab. 2007;51:197-203.

17. Caranti DA, de Mello MT, Prado WL, Tock L, Siqueira KO, de Piano A. Short- and long-term beneficial effects of a multidisciplinary therapy for the control of metabolic syndrome in obese adolescents. Metabolism. 2007;(56):1293-300.

18. Alcantara VM, Oliveira LC, Rea RR, Suplicy HL, Chautard-FreireMaia EA. Butyrylcholinesterase activity and metabolic syndrome in obese patients. Clin Chem Lab Med. 2005;43(3):285-8.

19. Kalman J, Juhasz A, Rakonczay Z, Abraham G, Zana M, Boda K, et al. Increased serum butyrylcholinesterase activity in type Ilb hyperlipidaemic patients. Life Sci. 2004;75(10):1195-204.

20. Clitherow JW, Mitchard M, Harper NJ. The possible biological function of pseudocholinesterase. Nature. 1963;199:1000-1.

21. Kutty KM, Huang SN, Kean KT. Pseudocholinesterase in obesity: hypercaloric diet induced changes in experimental obese mice. Experientia 37 (1981):1141-2.

22. Chu MI, Fontaine P, Kutty KM, Murphy D, Redheendran R. Cholinesterase in serum and low density lipoprotein of hyperlipidemic patients. Clin Chim Acta. 85(1978):55-9. 